\title{
Piceatannol protects against cerebral ischemia/reperfusion-induced apoptosis and oxidative stress via the Sirt1/FoxO1 signaling pathway
}

\author{
KAI-JIE WANG ${ }^{1 *}$, WEN-QIAN ZHANG ${ }^{1 *}$, JING-JING LIU $^{2}$, YING CUI $^{3}$ and JIAN-ZHONG CUI ${ }^{1}$ \\ ${ }^{1}$ Department of Neurosurgery, Tangshan Gongren Hospital; ${ }^{2}$ School of Clinical Medicine, North China University of \\ Science and Technology; ${ }^{3}$ Department of Neurology, Tangshan Gongren Hospital, Tangshan, Hebei 063000, P.R. China
}

Received June 20,2020; Accepted September 22, 2020

DOI: $10.3892 / \mathrm{mmr} .2020 .11618$

\begin{abstract}
Reperfusion is a critical therapeutic intervention used following acute ischemic stroke; however, it may cause cerebral ischemia/reperfusion injury (CIRI) and aggravate brain damage. Piceatannol (Pic), a hydroxylated analog of resveratrol, has been reported to exhibit anti-inflammatory effects. However, the detailed molecular mechanisms and its effects on CIRI have not been sufficiently assessed, and, to the best of our knowledge, current methods of prevention of CIRI are limited. The aim of the present study was to investigate the effects of Pic on improving neurological function in a mouse model of CIRI. For the animal experiments, 8 -week-old C57BL/6 mice were raised and randomly grouped, and an in vivo model of CIRI was established. Mice were administered a low $(10 \mathrm{mg} / \mathrm{kg} /$ day $)$ or high-dose $(20 \mathrm{mg} / \mathrm{kg} / \mathrm{d})$ of Pic $1 \mathrm{~h}$ after CIRI orally and once daily for the next 6 days. Neurological dysfunction was assessed using a modified neurological severity score and a rotarod test 1 week after CIRI establishment, and the cognitive status of the mice was assessed using a Morris water maze. Hematoxylin and eosin staining was used to evaluate the histopathological changes. The expression levels of sirtuin 1 (Sirt1), FoxO1, cleaved caspase-3 (CC-3), Bax and Bcl-2 were measured using western blotting. Intracellular reactive oxygen species (ROS) generation, antioxidant enzymes [superoxide dismutase, glutathione (GSH) peroxidase and catalase] and non-enzymatic antioxidants (GSH) were also detected using spectrophotometry. After inhibition of the Sirt1/FoxO1 pathway, a TUNEL assay was used for the detection of apoptotic cells in vitro and in vivo. The co-localization
\end{abstract}

Correspondence to: Dr Jian-Zhong Cui, Department of Neurosurgery, Tangshan Gongren Hospital, 27 Wenhua Road, Lubei, Tangshan, Hebei 063000, P.R. China

E-mail: jianzhongcuidoctor@163.com

*Contributed equally

Key words: ischemia/reperfusion injury, piceatannol, sirtuin 1, oxidative stress, apoptosis of neuron-specific nuclear protein and CC-3 was assessing using immunofluorescent staining. Pic improved neurological functions and ameliorated hippocampal neuronal pathology following CIRI. In addition, the expression levels of CC-3 and Bax and intracellular ROS levels were increased, while levels of antioxidant and non-enzymatic enzymes were decreased in the mouse model of CIRI. Low and high doses of Pic significantly decreased ROS production and the expression levels of apoptosis-related proteins, but increased antioxidant enzyme levels. However, a high-dose of Pic did not result in increased levels of non-enzymatic enzymes. Furthermore, low and high doses of Pic treatment significantly activated the Sirt1/FoxO1 pathway. Following inhibition of the Sirt1/FoxO1 pathway, the percentage of TUNEL-positive cells and expression of CC-3 were increased, and CC-3 was enriched in neurons. The antioxidant effects of Pic were blocked by inhibition of Sirt1 in vitro and in vivo. In conclusion, these results suggested that Pic may exert a neuroprotective effect against in hippocampal neurons via the Sirt1/FoxO1 pathway.

\section{Introduction}

Ischemic stroke refers to cerebral ischemia due to occlusion of a cerebral blood vessels, and has a high rate of mortality and morbidity (1). A cross-sectional study conducted in 2012-2013 demonstrated that the age-standardized incidence for stroke in China were 247/100,000 (95\% CI, 211-283), and ischemic stroke accounted for $\sim 60 \%$ among new-onset stroke patients (2). According to the 'China Health and Family Planning Statistical Yearbook 2016' (3), in 2015, the mortality of ischemic stroke was about 46.99 in 100,000 in the rural areas and 41.82 in 100,000 in the urban areas of China. The hippocampal injury is aggravated due to the lack of blood flow and oxygen deprivation, and cerebral ischemia/reperfusion injury (CIRI) is caused by subsequent restoration of blood flow (4). The mechanisms underlying the occurrence of CIRI are complicated and involve interactions between multiple factors. For example, CIRI causes tissue hypoxia, leading to reduced mitochondrial ATP synthesis, ion channel disorder, impaired enzyme activity and redox imbalance. These effects in turn may affect brain tissue oxygen uptake (5). In addition, the pathophysiological processes of CIRI are 
complicated, involving oxidative stress, inflammation, apoptosis and excitotoxicity (6-8). With the interruption in cerebral blood flow, accumulation of glutamate (Glu) is observed, and Glu-induced excitotoxicity involves consumption of intracellular glutathione (9). Along with the depletion of the endogenous antioxidant glutathione, excessive reactive oxygen species (ROS) accumulation can occur, resulting in increased oxidative stress (10). Severe oxidative stress may induce DNA damage and cell apoptosis, which affects neurological function (9). Therefore, the development of novel and effective antioxidants or therapeutic strategies to maintain the cellular redox homeostasis is urgently required.

Piceatannol (Pic), a natural stilbene found primarily in seeds, wines and fruits (11), has been reported to exhibit multiple protective effects on a variety of diseases, such as metabolic cardiomyopathy and ischemic cardiomyopathy (12,13). An increasing number of studies have revealed that Pic serves a crucial role in regulating a variety of physiological processes due to its anti-inflammatory capacity and potent antioxidant properties $(14,15)$. Additionally, Pic has been shown to participate in a variety of physiological and pathophysiological processes through the modulation of a range of pathways, which enables cells to respond to a diverse array of stimuli. Pic inhibited oxidative stress by activating the PI3K/Akt pathway, ultimately alleviating macular retinal pigment epithelial cells degeneration in mice in age-related macular degeneration (11). Moreover, Pic alleviated inflammation and oxidative stress by regulating the $\mathrm{Nrf} 2 / \mathrm{HO}-1$ and $\mathrm{NF}-\kappa \mathrm{B}$ pathways in diabetic cardiomyopathy (13).

The sirtuin 1 (Sirt1)/FoxO1 pathway is a vital signaling pathway considered to be involved in regulating oxidative stress, the inflammatory reaction and apoptosis (16). A recent in vitro study observed that activation of the Sirt1/FoxO1 pathway can alleviate neuron injury in an oxygen-glucose deprivation/reperfusion model, which may be due to the antioxidant activity and anti-apoptotic effects of the downregulation of this pathway (17).

Previous studies have reported that the beneficial effects of Pic may be due to the action of Sirt1, which contributes to upregulation of the antioxidative and anti-apoptotic factors against early-stage nephropathy and hepatic inflammation $(18,19)$. Although Pic has been demonstrated to exert protective properties in several cell types and different disease processes, such as diabetic cardiomyopathy and diabetic cardiomyopathy $(12,13)$, to the best of our knowledge, no studies have further examined its effects on CIRI, as well as the potential underlying molecular mechanisms. Furthermore, neuronal apoptosis, which has also been proposed as an important mechanism affecting neurologic function, may serve an important role in the pharmacodynamic processes (20). In combination with other evidence, the present study aimed to investigate the antioxidative stress damage and anti-apoptotic capability of Pic in vitro and in vivo, as well as to identified the possible involvement of certain signaling pathways.

\section{Materials and methods}

Animals and CIRI model. The animal experiments were approved by the Ethics Committee of Tangshan Gongren Hospital (approvalno.GRYY-LL-2019-13).Fortheexperiments, male C57BL/6 mice (age, 8 weeks; weight, 20-25 g; 60 mice in total) were obtained from the Experimental Animal Center, North China University of Science and Technology. The mice had free access to food and water throughout the entirety of the trial. The housing conditions were as follows: $20-24^{\circ} \mathrm{C}$, $12 \mathrm{~h}$ light/dark cycle and $50 \pm 10 \%$ humidity.

Mice underwent a middle-cerebral artery occlusion (MCAO) model of CIRI as previously described (21). Briefly, mice were anesthetized via intraperitoneal injection of sodium pentobarbital $(50 \mathrm{mg} / \mathrm{kg})$. During this stage, the body temperature of mice was maintained at $36.5-37.5^{\circ} \mathrm{C}$. Regional cerebral blood flow (rCBF) of each mouse was monitored using a Laser-Doppler flowmeter (Moor Instruments, Ltd.). A right side incision in the mouse was performed and the carotid arteries were separated, including the common carotid artery (CCA), external carotid artery and internal carotid artery (ICA). After carefully ligating the right CCA with a microclip, a silk thread was inserted into the stump of the ICA. The monofilament was then advanced $\sim 12 \mathrm{~mm}$ so that its distal end came to rest across the origin of the MCA. In order to minimize the differences in CIRI degree, performance of the MCAO surgery was regarded as successful if rCBF was decreased abruptly by $70-80 \%$. After obturating blood flow for $60 \mathrm{sec}$, the monofilament was then removed to re-establish carotid blood flow. Apart from Laser Doppler flowmetry, Longa's neurological scoring (22) was used to determine whether the MCAO model was successfully established. The animals with a score of 1-3 after MCAO were included for analysis. Given the inconsistencies between vascular occlusion degree and CIRI symptoms, mice with no or slight neurological deficit were excluded. The Sham group underwent only dissociation of carotid arteries without inserting a monofilament.

During the course of establishment of the CIRI model, mortality occurred in two mice due to disease conditions. The mortality was lower compared with previous estimates, which reported mortality rates up to $\sim 10 \%$ (21). After the model was successfully established, MCAO mice were randomly divided into CIRI, low-dose Pic and high-dose Pic groups using a random-number table, with 14 to 15 mice in each group.

Experimental groups and treatment. C57BL/6 J mice were randomly divided into four groups: Sham, CIRI, low (L)-dose Pic treatment $(10 \mathrm{mg} / \mathrm{kg} /$ day $)$ and high $(\mathrm{H})$-dose Pic treatment (20 mg/kg/day). Pic was purchased from Selleck Chemicals (cat. no. S3026) and suspended in $0.3 \mathrm{ml} 0.5 \%$ carboxymethyl cellulose solution prior to use. Mice were orally administered $\mathrm{L}$-and $\mathrm{H}$-doses of Pic $1 \mathrm{~h}$ after establishment of CIRI, and once daily for the next 6 days. Mice in the Sham and CIRI group were treated with $0.3 \mathrm{ml} 0.5 \%$ carboxymethyl cellulose solution by oral administration. After treatment, behavioral performance and pathological changes in brain hippocampal tissues of mice were detected using a sequential process.

Ex-527 (cat. no. E7034; Sigma-Aldrich; Merck KGaA), a Sirtl inhibitor, was used for subsequent experiments. An additional 60 mice were randomly assigned to the Sham, CIRI, CIRI + Pic treatment (20 mg/kg/day) and CIRI + Pic + Ex-527 groups. A total of $10 \mu \mathrm{g}$ Ex-527 was dissolved in a $5 \mu \mathrm{l}$ mixture of DMSO and normal saline before use. Subsequently, Ex-527 $(10 \mu \mathrm{g})$ was administered via intracerebroventricular injection $1 \mathrm{~h}$ before MCAO. Mice were placed in a stereotactic device 
after anesthetization. In accordance with the stereotaxic atlas of Paxinos and Watson (23), injections were made into the left lateral brain ventricle (lateral, 1.3; dorsoventral, 4.0; anteroposterior, -0.92 from Bregma and skull) using a stainless-steel guide cannula. When the guide cannula was $1-\mathrm{cm}$ above the skull, it was secured using dental acrylic. If no anomalies were found, a stainless-steel needle, with a diameter of $0.4 \mathrm{~mm}$, was inserted directly into the lateral ventricle along the guide cannula. Mice in the Sham and CIRI group were treated with $0.3 \mathrm{ml}$ of $0.5 \%$ carboxymethyl cellulose solution by oral and $5 \mu \mathrm{l}$ mixture of DMSO and normal saline via intracerebroventricular injection.

Rotarod performance test. In order to measure the effects of the drugs on vestibulomotor function, an accelerating automated rotarod test (RotaRod Treadmill; TSE Technical and Scientific Equipment) was used. Each mouse performed three trials per day. Mice were placed on a rotating rod that accelerated from 5-40 rpm during the 5-min experimental trial. Data obtained before the onset of MCAO were regarded as baseline values. Assessment of motor function was obtained at days 1-5 after CIRI. As the rotation velocity increased, the average time until the mice fell twice from the rotating drum was recorded manually.

Morris water maze (MWM) test. In order to determine the spatial learning and memory ability of the mice, MWM test was performed 1 week after completion of Pic treatments. The mice were placed in a round water pool (diameter $=150 \mathrm{~cm}$; height $=50 \mathrm{~cm}$ ) and allowed to swim freely in the water for $5 \mathrm{~min}$ at the start of the positioning navigation test. A hidden platform was set in the central area of the target quadrant. At a fixed time of each training day, mice were placed inside the tank to locate the hidden platform. At a designated starting point in a different quadrant of the maze, each mouse was placed randomly into the water. If the mouse succeeded in mounting the hidden platform during the navigation test, the mouse would be manually placed on the platform for $15 \mathrm{sec}$ before proceeding to the next training session.

The spatial exploration test, in which the hidden platform was removed, was performed on the 5th day of the experiment. Mice were placed gently into the pool at the same, randomly chosen training points and the residence time of each mouse in the target quadrant was recorded as 'target quadrant time'. Performance was recorded using a video camera connected to tracking equipment from HVS Tracking System Ltd.

Neurological score. Modified neurological severity score (mNSS) grades the composite neurological function of mice on sensory, motor, reflex and beam balance tests. The mNSS score was assigned as previously described (24). In the present study, neurological functional measurement was performed on mice within 1 week after CIRI establishment using the modified mNSS. A higher score indicated worse neural defects (least severe $=0$; most severe $=18$ ).

Hematoxylin and eosin (H\&E) staining. Briefly, after anesthetization and transcardial perfusions, the mice were rapidly executed via decapitation and hemispheres were removed. After fixing using 5\% neutral buffered formalin for 7 days at room temperature, the tissues were sliced into 4- $\mu \mathrm{m}$ sections. The sample slices were stained with hematoxylin for $2 \mathrm{~min}$ and with eosin for $30 \mathrm{sec}$. An optical microscope (Olympus Corporation; magnification, $\mathrm{x} 400$ ) was used to detect the histological changes in the hippocampal tissues.

Western blotting. Hippocampal tissues were lysed using RIPA lysis buffer (Thermo Fisher Scientific, Inc.). Protein concentrations were determined using a BCA assay (OriGene Technologies, Inc.). A total of $25 \mu \mathrm{g}$ extracted protein was loaded on a $12 \%$ SDS-gel, resolved using SDS-PAGE and electrophoretically transferred to a PVDF membrane (Bio-Rad Laboratories, Inc.). The membranes were blocked using 5\% non-fat milk for $1.5 \mathrm{~h}$ at room temperature. Subsequently, the membranes were washed three times with $\mathrm{PBS} / 0.2 \%$ Tween-20 (PBST) for $3 \mathrm{~min}$ each time, and incubated overnight at $4^{\circ} \mathrm{C}$ with rabbit anti-mouse antibodies. The primary antibodies used were: Bcl-2 (cat. no. ab196495; 1:1,000; rabbit polyclonal; Abcam), Bax (cat. no. ab32503; 1:1,000; rabbit monoclonal; Abcam), CC-3 (cat. no. ab49822; 1:1,000; rabbit polyclonal; Abcam), Sirt1 (cat. no. ab189494; 1:1,000; rabbit monoclonal; Abcam), FoxO1 (cat. no. ab52857; 1:1,000; rabbit monoclonal; Abcam) and $\beta$-actin (cat. no. ab8227; 1:1,000; rabbit polyclonal; Abcam). After washing three times for 5 min using PBST, the PVDF membranes were incubated with IRDye ${ }^{\circledR} 800 \mathrm{CW}$ goat anti-rabbit IgG secondary antibody (cat. no. ab216773; 1:10,000; Abcam) at room temperature for $2 \mathrm{~h}$. Signals were visualized using enhanced chemiluminescent reagent (Bio-Rad Laboratories, Inc.). Densitometry analysis was performed using ImageJ (Image Lab 4.1; National Institutes of Health).

Reverse-transcription quantitative PCR (RT-qPCR). Cellular RNA extraction was performed using TRIzol ${ }^{\circledR}$ reagent (Invitrogen; Thermo Fisher Scientific, Inc.) according to the manufacturer's protocol. A PrimeScript ${ }^{\mathrm{TM}}$ RT reagent kit (cat. no. RR037A; Takara Bio, Inc.) was used for cDNA generation. The RT reactions were performed at $37^{\circ} \mathrm{C}$ for $15 \mathrm{~min}, 85^{\circ} \mathrm{C}$ for $5 \mathrm{sec}$, then $4^{\circ} \mathrm{C}$ for cooling. The RT-qPCR was conducted with the TB Green ${ }^{\circledR}$ Premix Ex Taq ${ }^{\mathrm{TM}}$ II kit (cat. no. RR820A; Takara Bio, Inc.) in a 20- $\mu$ l reaction containing $10 \mu \mathrm{l} 2 \mathrm{X}$ TB Green Premix Ex Taq II (Tli RNaseH Plus), $0.8 \mu \mathrm{l}$ forward primer $(10 \mu \mathrm{M}), 0.8 \mu \mathrm{l}$ reverse primer

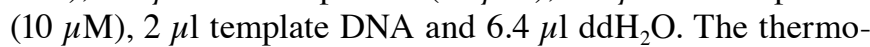
cycling conditions for RT were $37^{\circ} \mathrm{C}$ for $30 \mathrm{~min}$ followed by inactivation at $95^{\circ} \mathrm{C}$ for $1 \mathrm{~min}$. This was followed by 40 cycles of two-step PCR; $95^{\circ} \mathrm{C}$ for $18 \mathrm{sec}$ and $60^{\circ} \mathrm{C}$ for $55 \mathrm{sec}$, with a final extension step at $72^{\circ} \mathrm{C}$ for $2.5 \mathrm{~min}$, and subsequent holding at $4^{\circ} \mathrm{C}$. All experiments were repeated $\geq 3$ times. The sequences of the primers were: $\beta$-actin forward, 5'-TGACGT GGACATCCGCAAAG-3' and reverse, 5'-CTGGAAGGT GGACAGCGAGG-3'; CC-3 forward, 5'-GAGCACTGGAAT GTCATCTCGCTCTG-3' and reverse, 5'-TACAGGAAGTCA GCCTCCACCGGTATC-3'; Bax forward, 5'-GTTGCCCTC TTCTACTTTGC-3' and reverse, 5'-ATGGTCACTGTCTGC CATG-3'; Bcl-2 forward, 5'-GGTCCTCCAGTGGGTATT T-3' and reverse, 5'-TCCTCCTGAGACTGCCTTAT-3'; Sirt1 forward, 5'-AATCCAGTCATTAAACGGTCTACAA-3' and reverse, 5'-TAGGACCATTACTGCCAGAGG-3'; and FoxO1 forward, 5'-TTGGATTCCTGCCTGCTGAT-3' and reverse, 
5'-CGCTTCTTGGGTTGATCTAG-3'. $\beta$-actin was used as the internal control. The $2^{-\Delta \Delta \mathrm{Cq}}$ method was used to measure the relative gene expression (25).

ROS, antioxidant enzymes and non-enzymatic antioxidant detection. The procedures for detecting ROS production were based on the protocols provided by Applygen Technologies, Inc. (cat. no. C1300). After the hippocampal tissues were homogenized in $100 \mathrm{mM}$ sodium phosphate buffer (1:20), the homogenate was centrifuged at $1,000 \mathrm{x} \mathrm{g}$ for $10 \mathrm{~min}$ at $4^{\circ} \mathrm{C}$. A total of $10 \mu \mathrm{l} 1 \mathrm{mM}$ dichlorodihydrofluorescein diacetate was added to the supernatant. Fluorescence intensity was quantified after $30 \mathrm{~min}$ of incubation at $37^{\circ} \mathrm{C}$ using a fluorospectrophotometer (excitation, $500 \mathrm{~nm}$; emission, $525 \mathrm{~nm}$ ).

In the antioxidant enzymes system, superoxide dismutase (SOD) activity was determined spectrophotometrically using a SOD assay kit (Cayman Chemical Company) according to the manufacturer's protocol. At the beginning of the reaction, $20 \mu \mathrm{l}$ xanthine oxidase was injected into each well. The cells were incubated for $20 \mathrm{~min}$ at room temperature. According to the manufacturer's instructions (cat. no. A007-1-1; Nanjing Jiancheng Bioengineering Institute), catalase (CAT) activity was determined using the ammonium molybdate spectrophotometric method, which is based on the decreased absorbance due to consumption of $\mathrm{H}_{2} \mathrm{O}_{2}$ (26). Glutathione peroxidase (GSH-Px) activity was measured as described by Hafeman et al (27) in accordance with the manufacturer's instructions (cat. no. A005-1-2; Nanjing Jiancheng Bioengineering Institute). GSH-Px degrades $\mathrm{H}_{2} \mathrm{O}_{2}$ in the presence of GSH, which results in the decline of GSH. The remaining GSH reacts with 5,5'-dithiobis-2-nitrobenzoic acid (28). Absorbance was measured spectrophotometrically at $440-460 \mathrm{~nm}$ for SOD, $405 \mathrm{~nm}$ for CAT and $412 \mathrm{~nm}$ for GSH-Px at the end of reaction on a microplate reader (Infinite 200; Tecan Systems, Inc.).

For the non-enzymatic antioxidant system, homogenate preparation of hippocampal tissue was performed as described previously by Hissin and Hilf (28), and decreased GSH content was detected. After mixing with 5\% metaphosphoric acid, the supernatant was centrifuged at $2,000 \times \mathrm{g}$ for $2 \mathrm{~min}$ at $4^{\circ} \mathrm{C}$. Subsequently, Tris- $\mathrm{HCl}$ buffer ( $0.26 \mathrm{M} ; \mathrm{pH} 7.8), 0.65 \mathrm{~N} \mathrm{NaOH}$ and $\mathrm{O}$-phthalaldehyde $(1 \mathrm{mg} / \mathrm{ml})$ was added, and the supernatant was left in the dark for $15 \mathrm{~min}$ at $4^{\circ} \mathrm{C}$. A fluorescent microplate reader (Infinite 200; Tecan Systems, Inc.) was used to detect the fluorescent intensity with an excitation wavelength of $320 \mathrm{~nm}$ and emission wavelength of $420 \mathrm{~nm}$.

TUNEL staining for detection of neuronal apoptosis. Detection of neuronal apoptosis was performed as described previously (29). For brain tissue specimen collection from the cerebral ischemic area, mice were transcardially perfused with cold PBS followed by $4 \%$ paraformaldehyde in $0.1 \mathrm{M}$ phosphate buffer. Subsequently, brain tissues were extracted and fixed with $5 \%$ neutral buffered formalin for 7 days at room temperature. The specimens were dehydrated with gradient ethyl alcohol solutions and embedded in paraffin for $2 \mathrm{~h}$. TUNEL staining was performed using a TUNEL Apoptosis Assay Kit (cat. no. C1088; Beyotime Institute of Biotechnology). After sectioning, $5-\mu \mathrm{m}$ sections were deparaffinized and rehydrated. Following washing twice in $0.1 \mathrm{M}$ PBS $(2 \times 5 \mathrm{~min})$, the sections were incubated with $10 \mu \mathrm{g} / \mathrm{ml}$ Proteinase $\mathrm{K}$ working solution (pH 7.5-8.0) for $15 \mathrm{~min}$ at $37^{\circ} \mathrm{C}$. Then, tissues were rinsed again in PBS, and the slices were stained with green fluorescein-labeled dUTP solution for $10 \mathrm{~min}$ at room temperature. Finally, the sections were mounted with Vectashield ${ }^{\circledR}$ mounting medium containing DAPI for nuclear labeling for $5 \mathrm{~min}$ at room temperature (Vector Laboratories, Inc.). Using a fluorescence microscope (Olympus Corporation), TUNEL-positive cells that exhibited green fluorescent granules were detected. For quantification, five randomly selected fields of view were selected (magnification, $x 400$ ). The percentage of TUNEL positive cells per $\mathrm{mm}^{2}$ was determined. The apoptotic ratio is expressed as the percentage of the TUNEL-positive apoptotic neurons to the total number of DAPI stained neurons.

Immunofluorescence analysis. Hippocampal tissues were harvested and dehydrated with 15 and $30 \%$ gradient sucrose at $4^{\circ} \mathrm{C}$. Tissues were frozen, embedded in OCT compound (Sakura Finetek Japan, Co., Ltd.), then cut to $15-\mu \mathrm{m}$ frozen sections. After processing with $0.4 \%$ Triton X-100 for $10 \mathrm{~min}$, the frozen sections were blocked in $10 \%$ normal donkey serum (cat. no. ab138579; Abcam) for $1 \mathrm{~h}$ at room temperature. The sections were subsequently incubated with a mixture of primary CC-3 antibody (cat. no. ab49822; 1:1,000; Abcam) and neuronal nuclei (NeuN) monoclonal antibody (cat. no. ab177487; 1:1,000; Abcam) at $4^{\circ} \mathrm{C}$ overnight. Then, the cells were incubated for $1 \mathrm{~h}$ at $37^{\circ} \mathrm{C}$ with Alexa Fluor ${ }^{\circledR} 488$ goat anti-rabbit IgG secondary antibodies (cat. no. ab150077; 1:1,000; Abcam) the following day. Neurons were counterstained with DAPI for $10 \mathrm{~min}$ at room temperature. Confocal fluorescence images were acquired using an Olympus F1000 laser scanning confocal fluorescence microscope (magnification, $\mathrm{x} 400)$.

Primary hippocampal neuron culture. The embryos of 17-day pregnant Sprague-Dawley rats $(10 \mathrm{~g})$ were used for primary hippocampal neuron culture studies. The pregnant rats (3 months old, 280-350 g) were housed in individual cages and maintained on a 12 -h light $/ 12$-h dark cycle at $22 \pm 2^{\circ} \mathrm{C}$ and $65 \%$ humidity. All rats were allowed to take food and water ad libitum. The pregnant rats with embryos were anesthetized with isoflurane inhalation at dose of $4.5 \%$ for induction and $2.5 \%$ for maintenance. Pregnant rats were euthanized via cervical dislocation after the fetuses were removed. The fetuses were euthanized by $\mathrm{CO}_{2}$ inhalation and rapid decapitation (30). All efforts were made to minimize animal suffering. The skull was cut and opened by cutting along the midline with scissors. Following quick removal from the skull, hemispheres were separated and hippocampi were dissected on ice. Then, the hippocampi were cut into pieces and digested with trypsin (0.25\%; Gibco; Thermo Fisher Scientific, Inc.) at $37^{\circ} \mathrm{C}$ for $15 \mathrm{~min}$. The digested tissues were filtered and centrifuged at $1,500 \mathrm{x}$ g for $5 \mathrm{~min}$ at $4^{\circ} \mathrm{C}$, and the remaining cell pellet was resuspended with medium containing $92 \%$ Neurobasal medium, 5\% FBS (cat. no. 16140063; Gibco; Thermo Fisher Scientific, Inc.), 2\% B27, $1 \%$ Glu and $2 \mu \mathrm{l}$ gentamicin. Cells were seeded at a density of $1-5 \times 10^{5} / \mathrm{ml}$ in 6-well plates for $24 \mathrm{~h}$, arabinosylcytosin $(10 \mathrm{mg} / \mathrm{l})$ was added after $72 \mathrm{~h}$ at $37^{\circ} \mathrm{C}$ to prevent the growth of non-neuronal cells and the medium was subsequently half changed every 3 days. 
Glu-mediated excitotoxicity model and Pic treatment groups. After 7 days of culture, primary hippocampal neurons were washed with $\mathrm{Mg}^{2+}$-free extracellular solution followed by exposure to $100 \mathrm{mM}$ Glu (Sigma-Aldrich; Merck KGaA) for $5 \mathrm{~min}$ at room temperature in HEPES buffer containing $140 \mathrm{mM} \mathrm{NaCl}, 3.0 \mathrm{mM} \mathrm{KCl}, 2.0 \mathrm{mM} \mathrm{CaCl}_{2}, 1.0 \mathrm{mM} \mathrm{NaH}_{2} \mathrm{PO}_{4}$, $4.2 \mathrm{mM} \mathrm{NaHCO}, 10.0 \mathrm{mM}$ glucose and $10.0 \mathrm{mM}$ HEPES. Control group neurons were immersed in HEPES buffer for the same period. After washing, cultures were returned to the previous medium. Pic was dissolved in DMSO, such that the final DMSO concentration was $<0.1 \%$. The experimental groups were: Control, Glu, Pic + Glu and Pic + Glu + Sirt1small-interfering (si)RNA. Neurons transfected with control vector, were considered as the negative control group. An equivalent volume of DMSO was added at the same time point in the Control and Glu groups. A Cell Counting Kit-8 (CCK-8) assay (MedChem Express) was used to observe the optimal concentration and treatment period of Pic.

Cell viability assay. CCK-8 assays were used to investigate the optimal drug concentration of Pic for subsequent experiments, based on the cell viability of primary hippocampal neurons. Cell viability test was performed using a CCK-8 Kit according to the manufacturer's instructions (cat. no. CK04; Dojindo Molecular Technologies, Inc.). Briefly, following Glu treatment, primary hippocampal neurons were plated at a density of $1 \times 10^{4}-10^{5}$ cells/well in a 96 -well plate in triplicate. Neurons were cultured in a $\mathrm{CO}_{2}$ incubator at $37^{\circ} \mathrm{C}$ for $24 \mathrm{~h}$, and treated with different concentrations of Pic $(0.01,0.1,1,10$ or $100 \mathrm{mM})$ at $37^{\circ} \mathrm{C}$ for 12,24 or $48 \mathrm{~h}$. Subsequently, $10 \mu \mathrm{lCCK}-8$ solution was added to each well for $2 \mathrm{~h}$. Cell viability was analyzed using spectrophotometry at $450 \mathrm{~nm}$ in a microplate reader.

Transfection of siRNA. Neurons were grown in 6-well plates for $24 \mathrm{~h}$ and then transfected with Sirt1-siRNA (50 $\mathrm{nM})$ for $12 \mathrm{~h}$ using Lipofectamine $2000^{\mathrm{TM}}$ (Invitrogen; Thermo Fisher Scientific, Inc.). Cells were transfected for $48 \mathrm{~h}$, then used for subsequent experiments. Sirt1-specific siRNA and negative control (NC) siRNA were synthesized by Shanghai GenePharma Co., Ltd. Sirt1-siRNA sequences synthesized for rat neurons were as follows: Sense, 5'-GCGAUGUUAUAA UUAAUGAtt-3' and antisense, 5'-UCAUUAAUUAUAACA UCGCag-3'. Neurons treated with NC siRNA were used as the NC group. The NC forward sequence used was 5'-UUC UCCGAACGUGUCACGUTT-3', and reverse sequence was 5'-ACGUGACACGUUCGGAGAATT-3'. The transfection effect of siRNA was confirmed western blot. The molecular mass of Sirt1 was determined by running purified protein on $12 \%$ SDS-PAGE with standard molecular weight protein marker (Bio-Rad).

Statistical analysis. Data are presented as the mean $\pm \mathrm{SD}$, and were analyzed using SPSS version 23.0 (IBM, Corp.). Each experiment was performed a minimum of three times. Results of the rotarod performance test, mNSS and oriented navigation trials in MWM test were analyzed using a two-way mixed model ANOVA with Sidak's post hoc test. All other data were analyzed using a one-way ANOVA with Tukey's post hoc test for multiple comparisons. $\mathrm{P}<0.05$ (two-sided) was considered to indicate a statistically significant difference.

\section{Results}

Pic administration ameliorates CIRI-induced neurological deficits. The rotarod performance test, MWM test and mNSS provide a more synthetic evaluation of neurological functions following CIRI (31). The rotarod latencies of the CIRI mice were significantly shorter compared with that of the mice in the Sham group $(\mathrm{P}<0.05$; Fig. 1A), whereas a L-dose of Pic significantly increased rotarod latencies compared with the CIRI group $(\mathrm{P}<0.05)$. There was a significant difference in rotarod latencies between the Pic-L group and Pic-H group $(\mathrm{P}<0.05)$. mNSS were judged 1 week after CIRI. Mice with CIRI demonstrated impaired motor function compared with the Sham group $(\mathrm{P}<0.05$; Fig. $1 \mathrm{~B})$. By comparison with the CIRI group, Pic treatment significantly decreased the mNSS and mice exhibited improved neural function in a dose-dependent manner. Compared with the Sham group, CIRI led to notable learning and memory deficits (Fig. 1C); however, L-dose Pic treatment shortened the escape latency after the onset of CIRI $(\mathrm{P}<0.05)$. Mice in the Pic-H group exhibited a significantly improved performance in the positioning voyage test $(\mathrm{P}<0.05)$.

After the 4-day training period, the platform was moved away. As illustrated in Fig. 1D, a significant decline in the target quadrant time was observed in the CIRI group compared with the Sham group, while an increase in target quadrant time was observed in the L-dose Pic treatment groups compared with the CIRI group $(\mathrm{P}<0.05)$. The performance of mice in the L-dose Pic treatment was improved, compared with the low-dose group $(\mathrm{P}<0.05)$. However, there were no significant differences in swim speed during the space exploration trials (Fig. 1E).

Pic attenuates pathological injury caused by CIRI. Structural changes in hippocampal neurons was observed via H\&E staining. Hippocampal tissues in the Sham group demonstrated normal histology with palely stained neuronal cells and round nuclei (Fig. 2A). Compared with the Sham group, various histopathological changes were observed in the CIRI group, such as nuclear pyknosis, a decrease in neuronal numbers and neuron atrophy (Fig. 2B). When mice were treated with a L-dose of Pic, the number of hippocampal neurons increased and neuronal atrophy was alleviated, although it did not reach the level of the Sham group (Fig. 2C). Moreover, hippocampal tissues in the Pic-H treated group had less pathological impairment (Fig. 2D).

Pic reverses the increase of ROS levels and decreases the levels of antioxidant molecules. CIRI resulted in significantly increased ROS generation $(\mathrm{P}<0.01)$. By contrast, both L- and $\mathrm{H}$ dose Pic treatment attenuated CIRI-induced ROS increases (Fig. 3A). Moreover, there was a significant decline in the activity of SOD ( $\mathrm{P}<0.01$; Fig. 3B), CAT ( $<<0.01$; Fig. $3 \mathrm{C})$ and GSH-Px $(\mathrm{P}<0.05$; Fig. 3D) in the CIRI group compared with the Sham group. Of note, there was a rapid increase in cellular antioxidant enzyme activity in the Pic- $\mathrm{L}$ and $\mathrm{Pic}-\mathrm{H}$ treatment group in a dose-dependent manner $(\mathrm{P}<0.05)$.

As for the non-enzymatic antioxidants system, although Pic-L significantly increased GSH content compared with the CIRI group $(\mathrm{P}<0.05)$, no statistically significant difference was observed between the Pic-L and $\mathrm{Pic}-\mathrm{H}$ treatment group (P>0.05; Fig. 3E). 

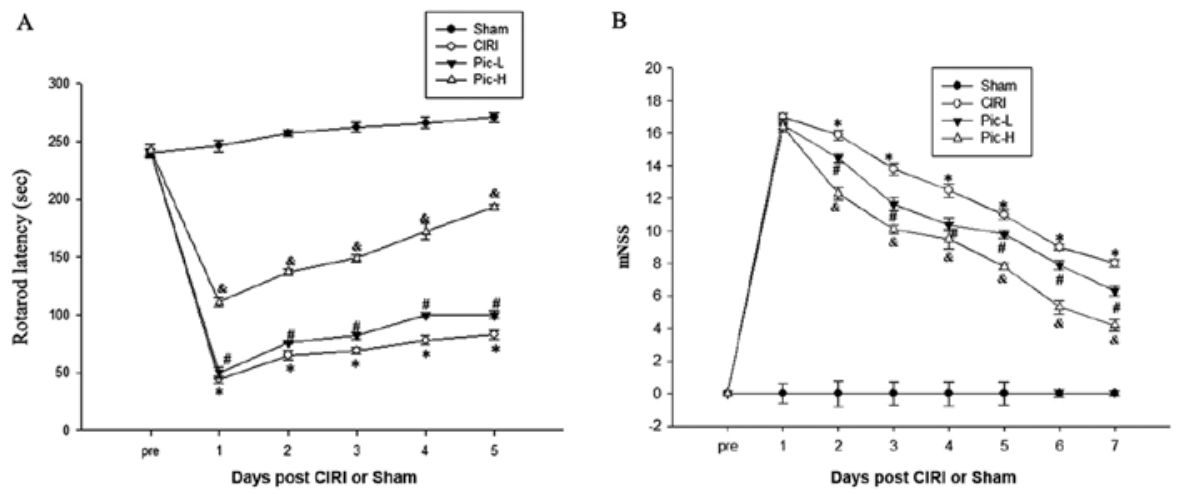

C
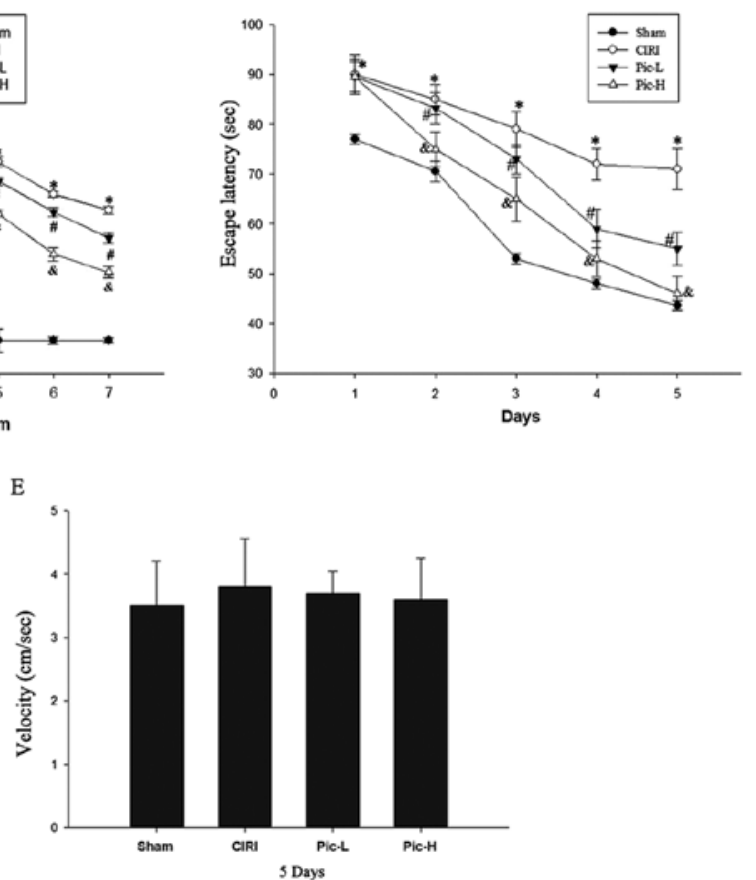

Figure 1. Neurological and cognitive function show improvement in Pic-treated mice following CIRI based on behavioral tests. (A) The vestibulomotor performance assessed via rotarod testing was significantly improved in the Pic-L and Pic-H group at 1-5 days after CIRI. (B) Recovery of neurobehavioral function based on mNSS. (C) Latency of locating the platform in the Morris water maze during the 4-day training period. (D) Time spent in target quadrant following removal of the platform. (E) There were no significant differences in swim speeds among groups. Data are presented as the mean \pm SD ( $=7$ per group). * $\mathrm{P}<0.05$ vs. Sham; ${ }^{\text {P }}<0.05$ vs. CIRI; ${ }^{\&} \mathrm{P}<0.05$ vs. Pic-L. $\mathrm{n}=13-15 /$ group. CIRI, cerebral ischemia-reperfusion injury; Pic, Piceatannol; L, low; H, high; $\mathrm{mNSS}$, modified neurological severity score.

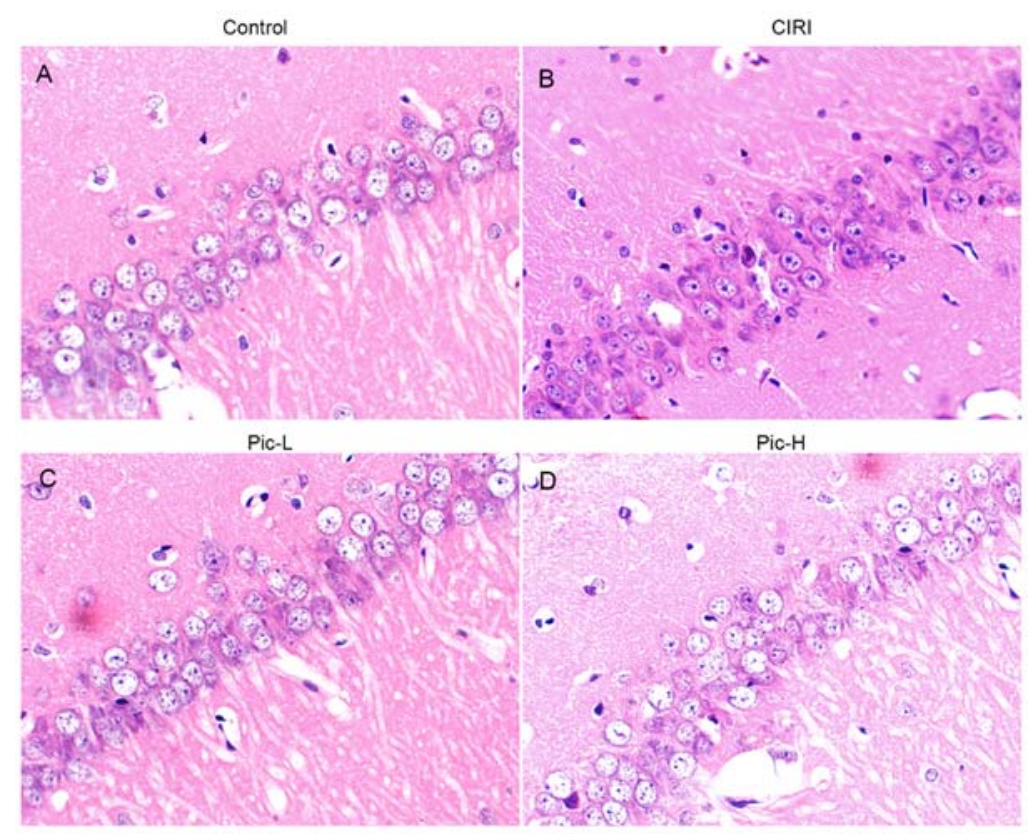

Figure 2. Histological analysis of the effects of Pic on neuronal injury induced by CIRI in mice. Hematoxylin and eosin staining was performed on tissue sections from the hippocampal Cornu Ammonis 1 regions (magnification, $x 400 ; n=5$ per group). (A) Neurons with normal histology were observed in the Sham group. (B) Altered neurons characterized by nuclear pyknosis, neurons decrease and neuron atrophy were found in the CIRI group. (C) Neuronal alterations were slightly eliminated in the Pic-L treatment group. (D) Neuronal alterations were significantly eliminated in the Pic-H treatment group. CIRI, Cerebral ischemia-reperfusion injury; Pic, Piceatannol; L, low; H, high.

Pic attenuates abnormalities in apoptosis-related markers caused by CIRI. In order to assess neuronal apoptosis in the hippocampus, the expression levels of CC-3, Bax and Bcl-2 were examined using western blotting. It was identified that there was a significant increase in the expression of $\mathrm{CC}-3$ $(\mathrm{P}<0.01$; Fig. $4 \mathrm{~A})$ and in the Bax/Bcl-2 ratio (P<0.01; Fig. 4B) in 

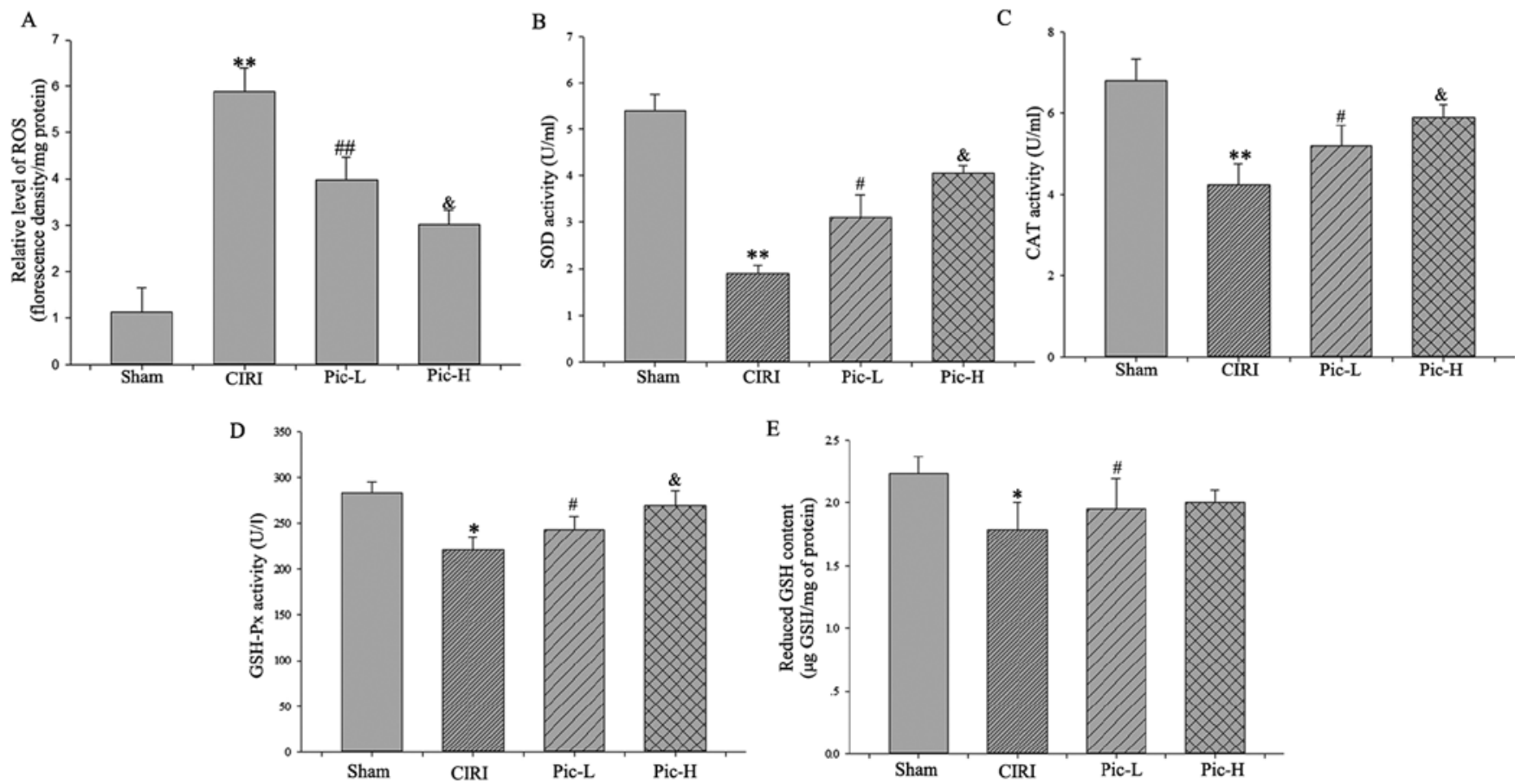

Figure 3. Pic reverses the increase of ROS and decrease in antioxidant molecules. (A) ROS production was evaluated as a marker of oxidative stress. Activities of (B) SOD, (C) CAT and (D) GSH-Px were detected to measure antioxidant enzymes system. (E) Content of decreased GSH was determined to

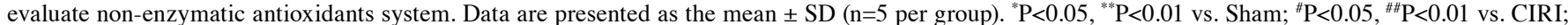
${ }^{\&} \mathrm{P}<0.05$ vs. Pic-L. CIRI, cerebral ischemia-reperfusion injury; Pic, piceatannol; ROS, reactive oxygen species; SOD, superoxide dismutase; CAT-Catalase; GSH-Px, glutathione peroxidase; GSH, glutathione; L, low; H, high.
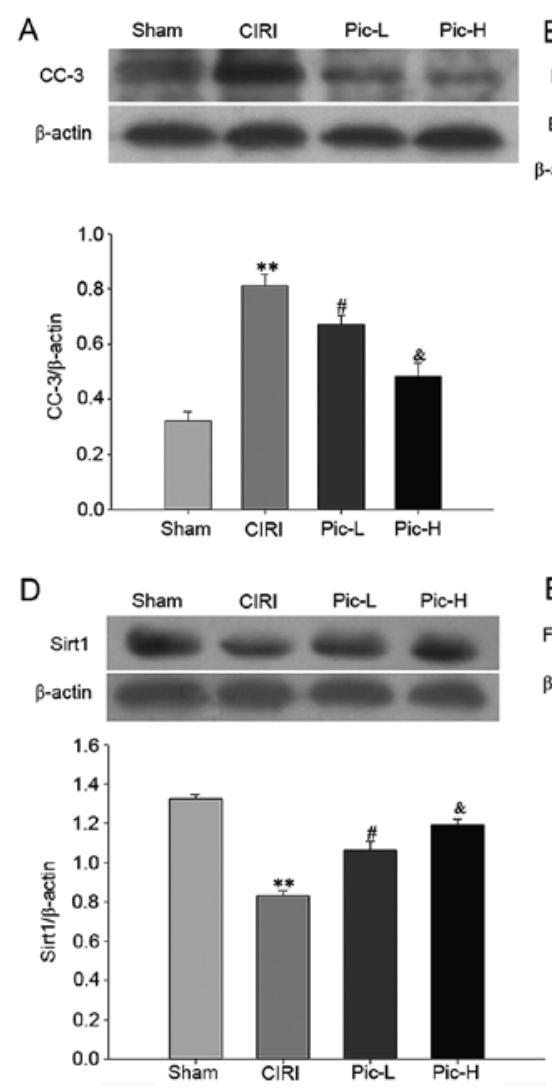
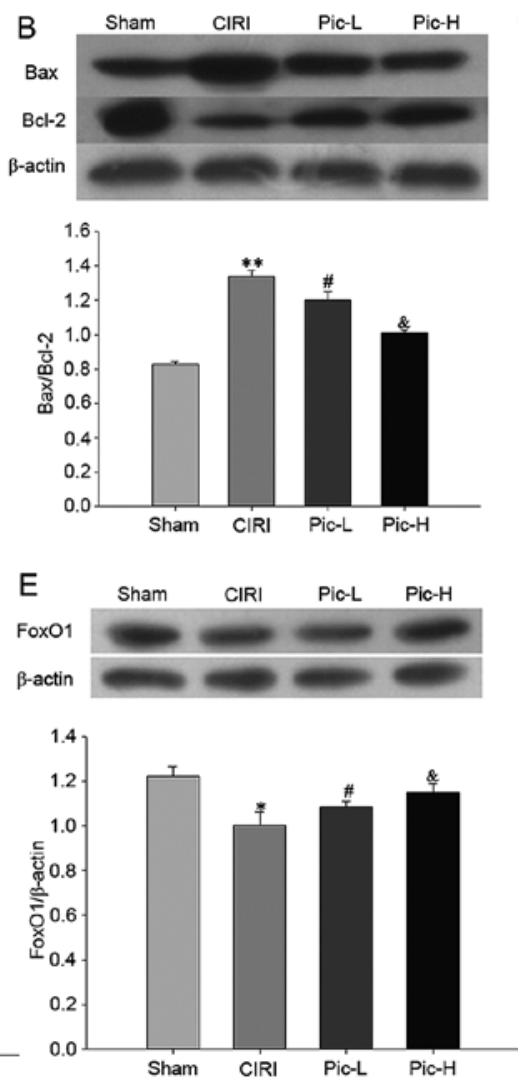

C

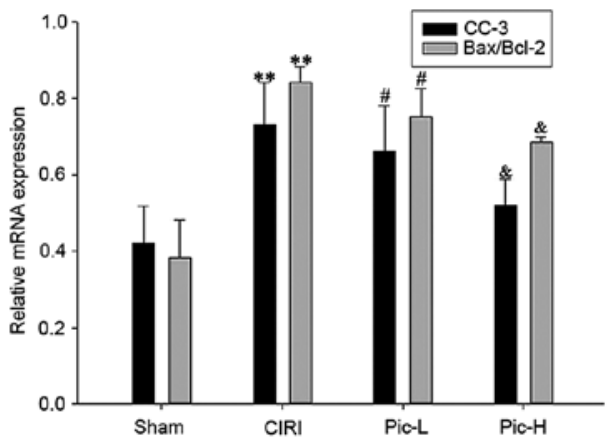

$\mathrm{F}$

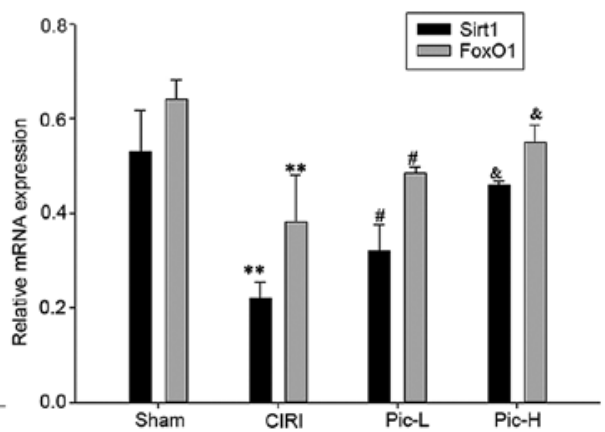

Figure 4. Administration of Pic attenuates CIRI-induced neuronal apoptosis and the deficit in the Sirt1/FoxO1 pathway. Hippocampal tissues from the mice were isolated immediately for western blot analysis, in order to detect the (A) CC-3, (B) Bax and Bcl-2 expression levels. (C) RT-qPCR results identified the relative mRNA expression levels of CC-3 and Bax/Bcl-2. Western blotting results of the relative expression levels of (D) Sirt1 and (E) FoxO1. (F) RT-qPCR revealed the relative expression of Sirt1 mRNA and FoxO1 mRNA. Data are presented as the mean $\pm \mathrm{SD}\left(\mathrm{n}=5\right.$ per group). ${ }^{*} \mathrm{P}<0.05,{ }^{* *} \mathrm{P}<0.01 \mathrm{vs}$. Sham; ${ }^{\#} \mathrm{P}<0.05$ vs. CIRI; ${ }^{\mathrm{P}} \mathrm{P}<0.05$ vs. Pic-L. CIRI, cerebral ischemia-reperfusion injury; Pic, piceatannol; Sirt1, sirtuin1; L, low; H, high; RT-qPCR, reverse transcription-quantitative PCR. 
A

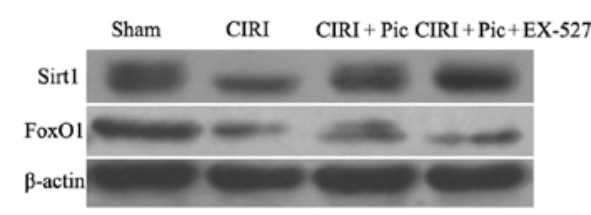

B

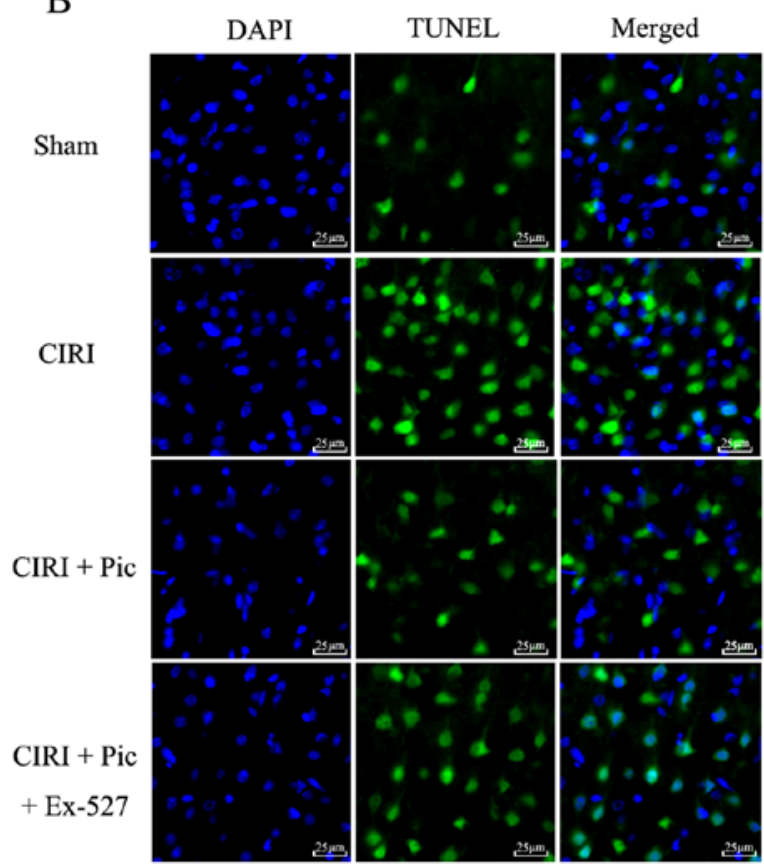

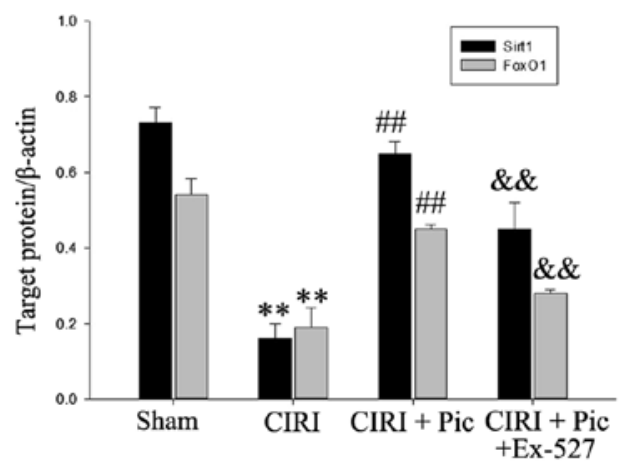

$\mathrm{C}$

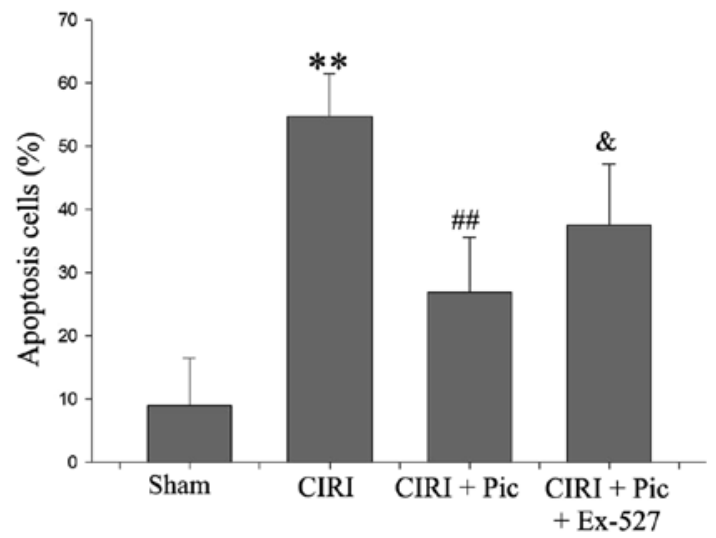

Figure 5. Representative images co-stained of DAPI (blue) and TUNEL (green) demonstrate the effect of Pic on neuron apoptosis after Sirt1/FoxO1 pathway inhibition. (A) Protein expression levels of Sirt1 and FoxO1 were inhibited with the addition of Ex-527. (B) TUNEL-positive cells were barely detected in the Sham group, but were widely distributed in CIRI group (magnification, $\mathrm{x} 400)$. Pic $(20 \mathrm{mg} / \mathrm{kg} / \mathrm{day})$ decreased the number of apoptosis cells, which was blocked by Ex-527. Scale bar, $25 \mu \mathrm{m}$. (C) Compared with the Sham group, apoptosis cells were significantly increased in CIRI group. Pic decreased the number of TUNEL-positive cells, whereas Ex-527 combined with Pic reversed this declining trend. Data are presented as the mean $\pm \mathrm{SD}\left(\mathrm{n}=5 \mathrm{per}\right.$ group). ${ }^{* *} \mathrm{P}<0.01 \mathrm{vs}$. Sham; ${ }^{\# \#} \mathrm{P}<0.01$ vs. CIRI; ${ }^{\&} \mathrm{P}<0.05$, ${ }^{\& \&} \mathrm{P}<0.01$ vs. CIRI + Pic. CIRI, cerebral ischemia-reperfusion injury; Pic, piceatannol; Sirt1, sirtuin1; L, low; H, high.

the CIRI group. A dose-dependent decrease in CC-3 expression and Bax/Bcl-2 ratio was observed following treatment with Pic (both doses $\mathrm{P}<0.05$ ). The CC-3 mRNA expression level and $\mathrm{Bax} / \mathrm{Bcl}-2$ mRNA ratio also demonstrated the same trend.

Pic attenuates the deficit in the Sirtl/FoxOl pathway. Significantly lower gene and protein expression levels of Sirtl and FoxO1 were observed in the CIRI group $(\mathrm{P}<0.01)$. Pic treatment significantly reversed this decrease in Sirtl and FoxO1 expression levels caused by CIRI. Moreover, the higher dose of Pic upregulated the Sirt1/FoxO1 signaling pathway to a greater degree compared with the Pic-L treatment group (all $\mathrm{P}<0.05$; Fig. 4D and E). The mRNA expression levels of Sirt1 and FoxO1 also demonstrated the same trend as the protein expression changes (Fig. 4F).

Pic decreases CIRI-induced apoptosis via activation of the Sirtl/FoxO1 pathway. The results of the inhibitory effect of Ex-527 on the Sirt1/FoxO1 pathway are presented in Fig. 5A. CIRI reduced the expression Sirt1/FoxO1 pathway-related proteins compared with Sham group. Upon addition of Pic, the Sirt1/FoxO1 pathway is activated. By contrast, Ex-527 counteracted the activation of Sirt1/FoxO1 pathway by Pic. The presence of TUNEL-positive cells was significantly increased in the CIRI group compared with the Sham group (Fig. 5B and C). Compared with CIRI group, the number of apoptotic hippocampal neurons was significantly decreased in the CIRI + Pic group $(\mathrm{P}<0.01$; Fig. $5 \mathrm{C})$. Furthermore, the proportion of apoptotic neurons was significantly increased by addition of Ex-527 $(\mathrm{P}<0.05)$, compared with the CIRI + Pic group.

Double immunofluorescent staining of CC-3 and NeuN indicated that Pic treatment not only decreased the protein expression of CC-3, but also significantly increased neuronal survival of the CIRI mice. However, after administration of Ex-527, apoptosis of hippocampal neurons increased compared with the CIRI + Pic group ( $\mathrm{P}<0.05$; Fig. 6).

Pic alleviates oxidative damage by modulating the Sirt1/FoxOI pathway. After inhibition of the Sirt1/FoxO1 pathway, the generation of ROS significantly increased compared with that 
A

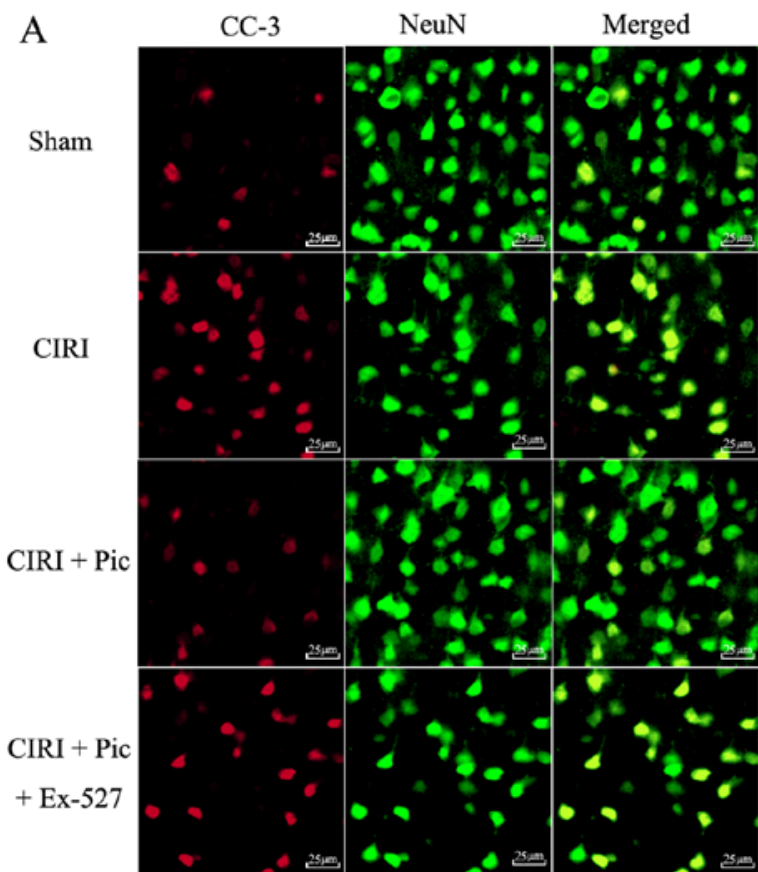

B

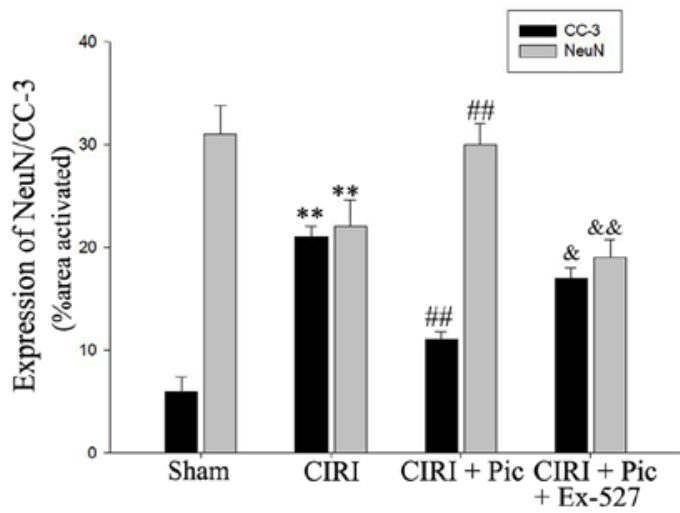

Figure 6. Immunofluorescence detection of neurons demonstrated that inhibition of the Sirt1/FoxO1 pathway with Ex-527 diminishes the protective effects of Pic on apoptosis neurons. (A) Results of the double immunofluorescent staining identified that CC-3 was co-localized with NeuN in the hippocampus. Magnification, $x 400$. (B) Quantification of fluorescence intensity. Data are presented as the mean \pm SD ( $=5$ per group). ${ }^{* *} \mathrm{P}<0.01$ vs. Sham; ${ }^{\#} \mathrm{P}<0.01 \mathrm{vs}$. CIRI; ${ }^{\&} \mathrm{P}<0.05,{ }^{\&} \mathrm{P}<0.01$ vs. CIRI + Pic. CIRI, cerebral ischemia-reperfusion injury; Pic, piceatannol; CC-3, cleaved caspase-3; NeuN, neuronal nuclei; Sirt1, Sirtuin1.

in the CIRI + Pic group $(\mathrm{P}<0.05$; Fig. 7A). Conversely, there was a significant decline in the SOD, CAT and GSH-Px activities following treatment with Ex-527 (P<0.05; Fig. 7B-D), suggesting that Pic upregulated cellular antioxidant enzymes activity via the Sirt1/FoxO1 pathway.

With regards to the non-enzymatic antioxidant system, the increased GSH content in the CIRI + Pic group was reversed by inhibition of the Sirt1/FoxO1 pathway using Ex-527 $(\mathrm{P}<0.05$; Fig. 7E).

Inhibiting the activity of the Sirt1/FoxO1 pathway reverses the effects of Pic on the apoptotic rate and antioxidant enzymes in primary hippocampal neurons. In the in vitro experiments, in order to evaluate the functional effects of Pic on neurons treated with or without Glu, the effect of different doses of Pic (0.01, $0.1,1,10$ or $100 \mathrm{mM}$ ) on cell viability was investigated. Glu significantly inhibited cell viability at all time points and all concentrations of Pic. Compared with the control group, 0.01, $0.1,1$ and $10 \mathrm{mM}$ PIC significantly promoted neuronal viability, irrespective of Glu treatment $(\mathrm{P}<0.05$; Fig. 8A-B). However, $100 \mathrm{mM}$ Pic significantly reduced cell viability compared with cells treated with $10 \mathrm{mM}$ Pic $(\mathrm{P}<0.05$; Fig. 8A and B). Thus, $10 \mathrm{mM}$ Pic was used for subsequent experiments. Within $24 \mathrm{~h}$, cell viability was increased in a time-dependent manner, but decreased after $24 \mathrm{~h}(\mathrm{P}<0.05$; Fig. 8C).

The silencing effect of Sirt1-siRNA is presented in Fig. 8D and E. The rate of apoptosis was defined as the number of TUNEL-positive cells divided by the number of DAPI stained cells. The results demonstrated that knockdown of Sirt1 significantly weakened the protective effects of Pic on hippocampal neurons from Glu-induced apoptosis (Fig. 8F and G). Compared with the Glu treated group, the activities of SOD and CAT were increased in the Pic + Glu group, and the expression of the antioxidant enzymes were decreased when Sirt1 expression was knocked down $(\mathrm{P}<0.05$; Fig. $8 \mathrm{H})$.

\section{Discussion}

Ischemic stroke is currently a major health concern that is considered a worldwide health issue. A large-scale survey in China in 2013 reported that the prevalence of stroke was $1.114 \%$, and cerebral ischemic stroke accounted for $\sim 70 \%$ (2). Cerebral artery occlusion may cause a sudden interruption of cerebral artery blood supply, leading to cerebral anoxia and ROS accumulation (6). Upon restoration of blood flow, cerebral oxidative stress results in further aggravation, leading to an imbalance between antioxidant defense mechanisms and oxidant production, thus causing a disturbance of cell-survival mechanisms and ultimately neurological damage $(32,33)$. In the present study, the effects of Pic against oxidative stress and neuronal apoptosis in a murine mouse model of CIRI were assessed, as well as the possible mechanisms and the involved signaling pathways.

Pic belongs to the natural polyphenolic compound family, and has been confirmed to be a naturally occurring hydroxylated analogue of resveratrol (34). Due to the similarity in the molecular structure with resveratrol, Pic was hypothesized to exert similar pharmacological properties. Previous studies reported that Pic possesses a multitude of biological properties, such as anti-cancer, antioxidative and hepato- and neuroprotective effects (35-37). It is now well established that cell viability increases in a dose-dependent manner when cells are treated with Pic following injury (38). Furthermore, Pic can exert its anti-oxidant effects via the upregulation of anti-oxidant enzymes such as SOD and CAT $(36,39)$. Pic is rapidly metabolized by the liver and is converted 

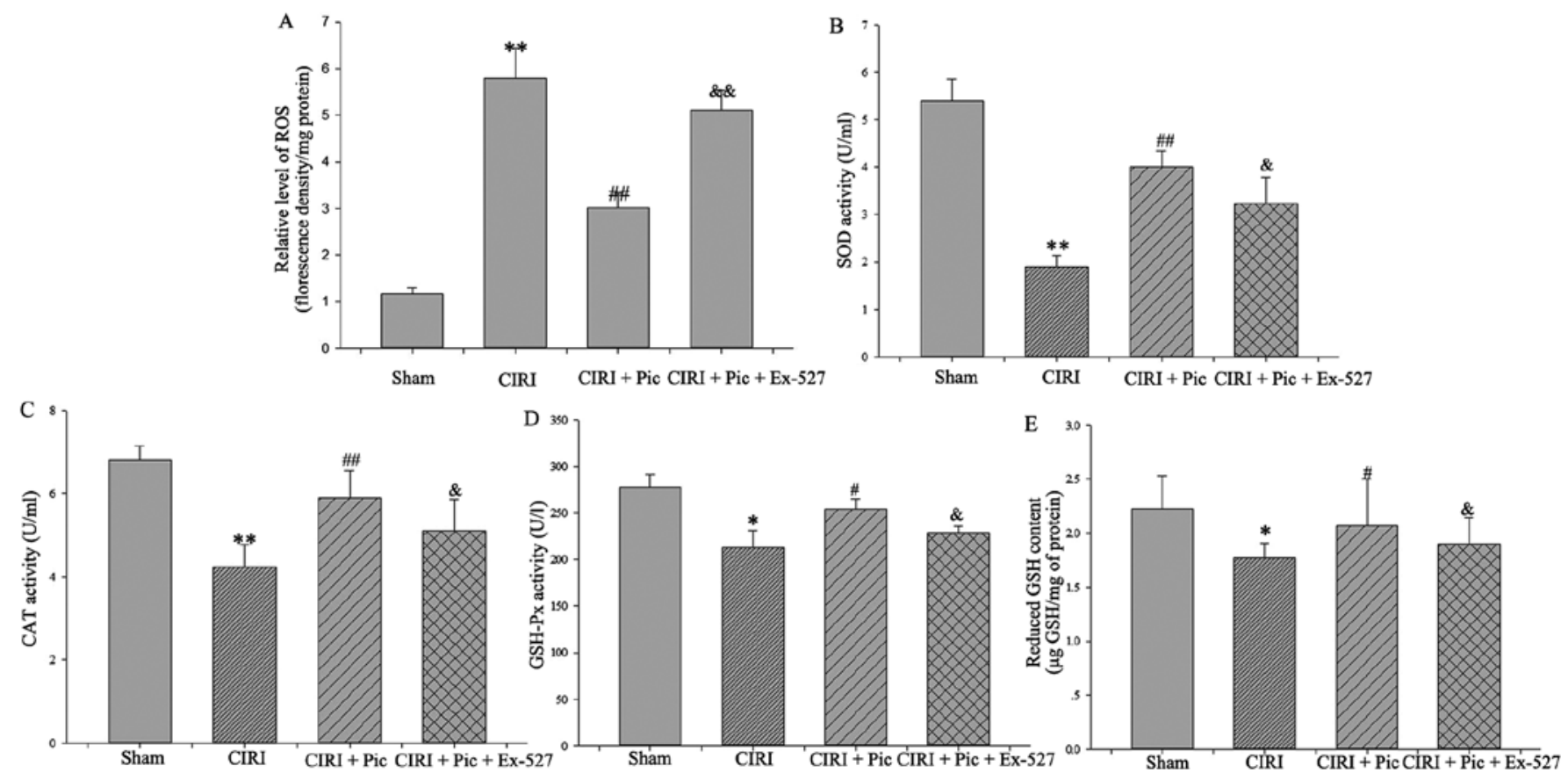

Figure 7. Inhibition of the Sirt1/FoxO1 pathway reverses the decrease of ROS and increase in antioxidant molecules. (A) Pic treatment caused a decrease of ROS production in the neurons, whereas inhibition of the Sirt1/FoxO1 pathway could significantly reverse Pic treatment-induced ROS reduction. Antioxidant enzymes systems, (B) SOD, (C) CAT and (D) GSH-Px, and the non-enzymatic antioxidants system, (E) reduced GSH, were also declined after inhibition of the Sirt1/FoxO1 pathway. Data are presented as the mean $\pm \mathrm{SD}$ ( $\mathrm{n}=5$ per group). ${ }^{*} \mathrm{P}<0.05,{ }^{* *} \mathrm{P}<0.01$ vs. Sham; ${ }^{\prime} \mathrm{P}<0.05,{ }^{\# \#} \mathrm{P}<0.01$ vs. CIRI; ${ }^{\text {\& }} \mathrm{P}<0.05$, \&\& $\mathrm{P}<0.01$ vs. CIRI + Pic. CIRI, cerebral ischemia-reperfusion injury; Pic, piceatannol; ROS, reactive oxygen species; SOD, superoxide dismutase; CAT, catalase; GSH, Px-Glutathione peroxidase; GSH, glutathione; Sirt1, Sirtuin1.

predominantly into a glucuronide conjugate (40). Thus, it was speculated that increasing the concentration of Pic appropriately would increase its antioxidant properties, and this was previously confirmed by Yokozawa and Kim (41).

The present study demonstrated that mice with CIRI exhibited an increase in ROS production, accompanied by a decrease in the expression of antioxidative enzymes. Pic treatment $(10 \mathrm{mg} / \mathrm{kg} /$ day $)$ decreased the generation of ROS, and this decline was significantly higher in the Pic-H treatment group. Moreover, SOD, CAT and GSH-Px activities, which are used as indicators of antioxidant capability, was determined in the present study. The activity of SOD, CAT and GSH-Px was markedly upregulated in both low dose and high dose Pic-treated mice; animal experiments performed by Wen et al (36) and Zhang et al (37) also presented the same results. However, it was identified that Pic upregulated GSH content in the CIRI mice, but this effect was not dose-dependent. Other study also reported the effects of Pic on non-enzymatic enzyme system, but did not determine whether these effects were dose-dependent (41). Therefore, further study is required to determine the potential molecular mechanisms to validate the current findings.

In addition to alleviating oxidative stress, Pic is reported to exert anti-apoptotic properties by downregulating CC-3, as well as maintaining the balance between the apoptosis-related genes Bax and Bcl-2 (20). The present results suggested that low dose and high dose of Pic decreased the expression of CC-3 and Bax/Bcl-2 at the transcriptional level, but it did not reach the levels found in the sham group. Despite limited neuroprotection, Pic was able to exert a small but beneficial effect on neural survival. It has also been shown that caspases, such as C-9 and C-3, and poly (ADP-ribose) polymerase are activated by Pic in a dose-dependent manner $(20,42)$. The present results were consistent with previous study confirming the reliability of the effects of Pic (42). However, due to the different cell types and intervention methods used, there were some inconsistencies with prior studies. For instance, Song et al (43) revealed that Pic treatment was able to increase tumor apoptosis. In a model of transplanted mammary cancer, Pic treatment was able to increase the expression levels of Bax and $\mathrm{CC}-3$, but downregulate $\mathrm{Bcl}-2$ expression in tumor tissues (43). Increased numbers of tumor cells undergo apoptosis following Pic treatment, suggesting that Pic inhibits the breast tumor growth via the induction of apoptosis (43). Studies determining and targeting the potential mechanisms regulated by Pic with regards to cell survival are required to examine the differences between the present and previous studies.

As for exact mechanism via which Pic exhibits a positive role, numerous studies have shown that Pic activates specific signaling pathways to exhibit antioxidant activities and anti-apoptotic effects, such as the PI3K/Akt and nuclear factor, erythroid 2 like 2 /heme oxygenase 1 signaling pathways $(13,44)$. Due to the similarity in the structure to resveratrol, Pic was speculated to exhibit an active role via similar mechanisms. A previous study revealed that Pic and resveratrol both improved ischemia and anoxia via upregulating HIF-1 $\alpha$ expression, and downregulating c-Myc, egl-9 family hypoxia inducible factor 1 and $\beta$-catenin expression levels via the activation of Sirt1 (45). The NAD ${ }^{+}$-dependent protein deacetylase Sirt1 serves a critical role in the inhibition of oxidative stress and suppression of apoptosis along with downstream signaling of the FoxO1 pathway (46). In the present study, Pic enhanced antioxidant activity and suppressed apoptosis in a dose-response manner, 


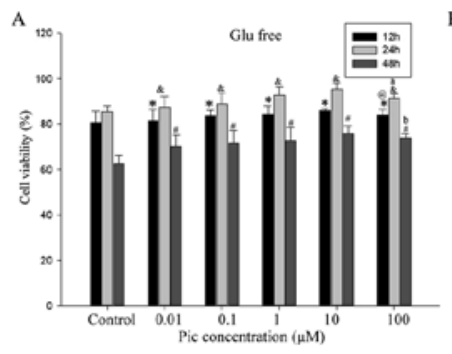

E
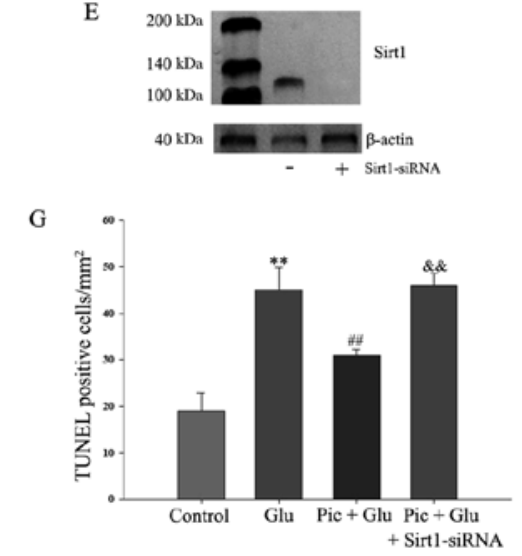

$\mathrm{H}$

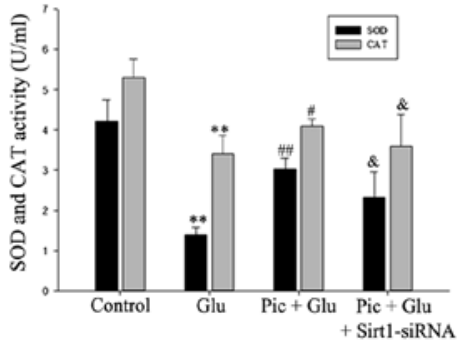

G

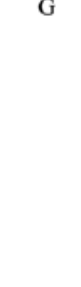

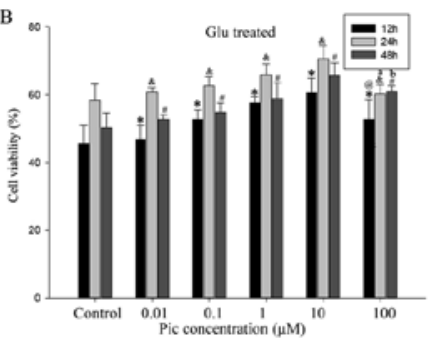
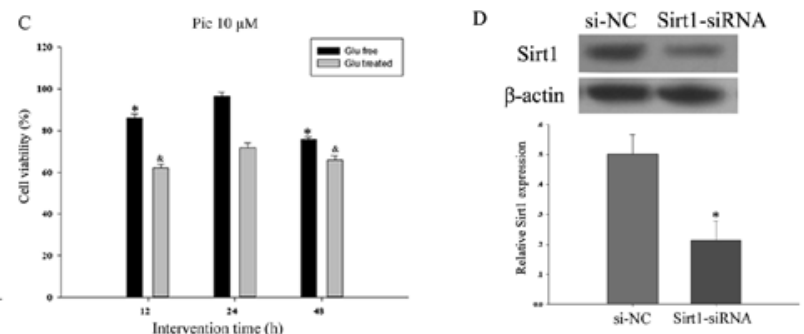

F
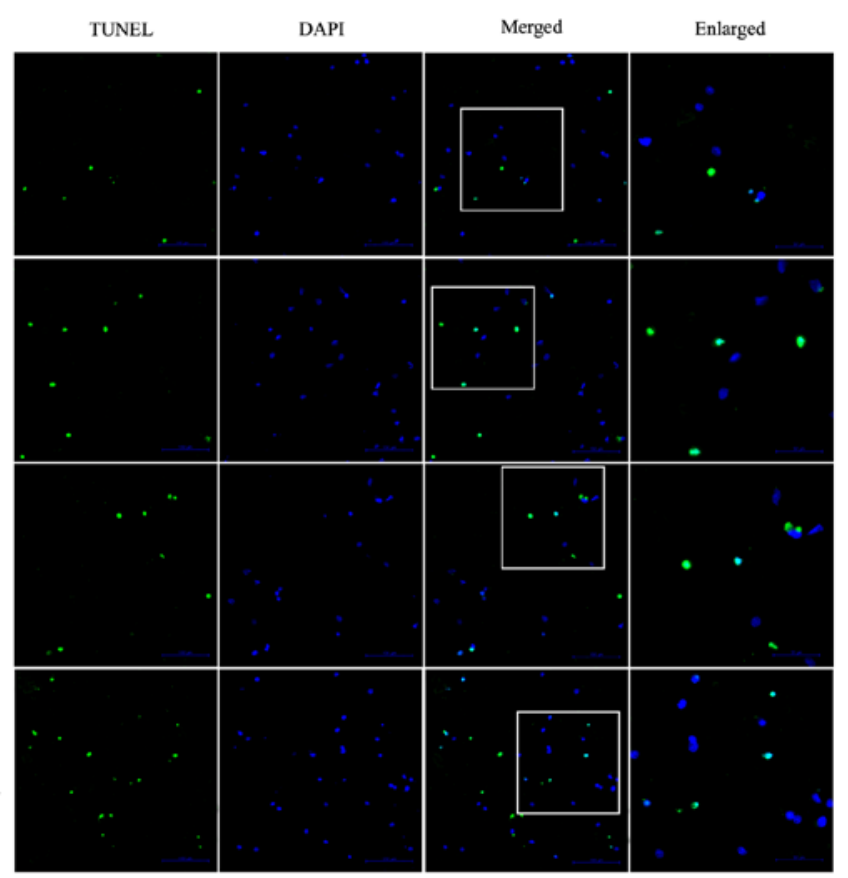

Figure 8. Effect of Pic on the apoptotic rate of the primary hippocampal neurons after Sirt1-siRNA treatment. First, cells were treated with different concentrations $(0.01,0.1,1,10$ and $100 \mathrm{mM})$ of Pic under Glu free or Glu treated conditions. Cell viability treated (A) with and (B) without Glu at 12,24 and $48 \mathrm{~h}$ with different Pic concentrations. ${ }^{*} \mathrm{P}<0.05$ vs. control group at $12 \mathrm{~h} ;{ }^{\&} \mathrm{P}<0.05$ vs. control group at $24 \mathrm{~h} ;{ }^{\#} \mathrm{P}<0.05$ vs. control group at $48 \mathrm{~h}$; ${ }^{\circledR} \mathrm{P}<0.05 \mathrm{vs}$. Pic at $10 \mathrm{mM}$ at $12 \mathrm{~h} ;{ }^{a} \mathrm{P}<0.05 \mathrm{vs}$. Pic at $10 \mathrm{mM}$ at $24 \mathrm{~h} ;{ }^{\mathrm{b}} \mathrm{P}<0.05$ vs. Pic at $10 \mathrm{mM}$ at $48 \mathrm{~h}$. (C) Cell viability under the Pic concentration of $10 \mathrm{mM}$ with scheduled duration of trial treatment. * $\mathrm{P}<0.05$ vs. Glu free condition at $24 \mathrm{~h} ;{ }^{\&} \mathrm{P}<0.05 \mathrm{vs}$. Glu-treated condition at $24 \mathrm{~h}$. (D) Western blotting results demonstrating (E) the silencing effect of Sirt1-siRNA. "P<0.05 vs. si-NC. (F) TUNEL-stained green cells indicated apoptosis-positive cells, DAPI-stained blue as nucleated cells and Merge stained with TUNEL and DAPI. Magnification, x200. (G) Quantification of TUNEL positive cell ratio in different groups. (H) Inhibiting the activity of the Sirt1/FoxO1 pathway can reverse the effect of Pic on the activities of SOD and CAT. Data are presented as the mean \pm SD ( $=5$ per group). ${ }^{*}<0.05$, ${ }^{* *} \mathrm{P}<0.01$ vs. Control; ${ }^{\#} \mathrm{P}<0.05,{ }^{\# \#} \mathrm{P}<0.01$ vs. Glu; ${ }^{\&} \mathrm{P}<0.05$, ${ }^{\&} \mathrm{P}<0.01$ vs. Pic + Glu. Glu, glutamate; CIRI, cerebral ischemia-reperfusion injury; Pic, piceatannol; SOD, superoxide dismutase; CAT, catalase; NC, negative control; siRNA, small interfering RNA; Sirt1, Sirtuin1.

and the activity of the Sirt1/FoxO1 signaling pathway was upregulated simultaneously. When cells were treated with Ex-527 or Sirt1-siRNA, an increase in the number of apoptotic cells and increased oxidative stress was observed. These results suggested that the Sirt1/FoxO1 signaling pathway may be associated with the antioxidant potency and anti-apoptotic abilities of Pic on hippocampal neurons.

In addition to the aforementioned cytoprotective effects, the administration of Pic exerts several other effects on cellular biological processes, such as anti-inflammatory effects (19), metabolic improvement (47) and astrocyte differentiation (48). Several hypotheses have been suggested as underlying the effects of Pic on CIRI, such as inhibition of the activation of inflammatory pathway and innate immune system $(49,50)$. The presence of numerous hypotheses may be explained by the fact that the neuroprotective effects of Pic may be the result of multiple interactions. Therefore, additional studies using further comprehensive approaches are required to evaluate the underlying mechanisms that maintained neuronal homeostasis. Moreover, investigating other natural products with similar chemical structures may provide a more definitive conclusion. Whether Pic can also reverse anticoagulant, such as tissue plasminogen activator, induced toxicity at the subacute stage of ischemic stroke warrants further investigation. These findings would have an important clinical impact on the neuroprotection of patients undergoing intravenous thrombolysis with anticoagulants.

In conclusion, Pic treatment was capable of exerting neuroprotective effects that were largely due to the maintenance of the balance of the antioxidative/oxidative system, as well as the protection of hippocampal neurons from apoptosis. Furthermore, the Sirt1/FoxO1 signaling pathway was identified to be involved in both of these processes. The present study provides additional evidence that highlights the potential use of Pic as a clinically relevant therapeutic for ischemic stroke. 


\section{Acknowledgements}

Not applicable.

\section{Funding}

The present study was supported by a Project of Key Medical Science Research Program of Hebei Province (grant no. 20171355).

\section{Availability of data and materials}

The datasets used and/or analyzed during the current study are available from the corresponding author on reasonable request.

\section{Authors' contributions}

KJW, WQZ and JZC conceived and designed the study. JJL and YC were responsible for assessment and accreditation of laboratory animal care. KJW, WQZ and YC were responsible for data analysis. JJL and JZC performed histological and behavioral experiments. KJW, WQZ and JZC wrote the manuscript. JZC reviewed and edited the manuscript. All authors read and approved the final manuscript.

\section{Ethics approval and consent to participate}

All research was conducted with the approval of the Ethics Committee of Tangshan Gongren Hospital (clearance no. GRYY-LL-2019-13).

\section{Patient consent for publication}

Not applicable.

\section{Competing interests}

The authors declare that they have no competing interests.

\section{References}

1. Zerna C, Thomalla G, Campbell BCV, Rha JH and Hill MD: Current practice and future directions in the diagnosis and acute treatment of ischaemic stroke. Lancet 392: 1247-1256, 2018.

2. Wang W, Jiang B, Sun H, Ru X, Sun D, Wang L, Wang L, Jiang Y, Li Y, Wang Y, et al: Prevalence, incidence, and mortality of stroke in China clinical perspective: Results from a nationwide population-based survey of 480687 adults. Circulation 135: 759-771, 2017.

3. China's Health and Family Planning Statistical Yearbook National Health and Family Planning Commission of the People's Republic of China. Peking: Peking Union Medical College Press, 2016.

4. Jean WC, Spellman SR, Nussbaum ES and Low WC: Reperfusion injury after focal cerebral ischemia: The role inflammation and the the rapeutic horizon. Neurosurgery 43: 1382-1396, 1998.

5. Wu M, Yiang GT, Liao WT, Tsai AP, Cheng YL, Cheng PW, Li CY and Li CJ: Current mechanistic concepts in ischemia and reperfusion injury. Cell Physiol Biochem 46: 1650-1667, 2018.

6. Yang Q, Huang Q, Hu Z and Tang X: Potential neuroprotective treatment of stroke: Targeting excitotoxicity, oxidative stress, and inflammation. Front Neurosci 13: 1036, 2019.

7. Nakka VP, Gusain A, Mehta SL and Raghubir R: Molecular mechanisms of apoptosis in cerebral ischemia: Multiple neuroprotective opportunities. Mol Neurobiol 37: 7-38, 2008.
8. Candelario-Jalil E: Injury and repair mechanisms in ischemic stroke: Considerations for the development of novel neurotherapeutics. Curr Opin Investig Drugs 10: 644-654, 2009.

9. Huang HL, Fang LW, Lu SP, Chou CK, Luh TY and Lai MZ: DNA-damaging reagents induce apoptosis through reactive oxygen species-dependent Fas aggregation. Oncogene 22: 8168-8177, 2003

10. Lin TC, Chen YR, Kensicki E, Li AYJ, Kong M, Li Y, Mohney RP, Shen HM, Stiles B, Mizushima N, et al: Autophagy: Resetting glutamine-dependent metabolism and oxygen consumption. Autophagy 8: 1477-1493, 2012.

11. Hao Y, Liu J, Wang Z, Yu LL and Wang J: Piceatannol protects human retinal pigment epithelial cells against hydrogen peroxide induced oxidative stress and apoptosis through modulating PI3K/Akt signaling pathway. Nutrients 11: 1515, 2019.

12. Boccellino M, Donniacuo M, Bruno F, Rinaldi B, Quagliuolo L, Ambruosi M, Pace S, De Rosa M, Olgaç A, Banoglu E, et al: Protective effect of piceatannol and bioactive stilbene derivatives against hypoxia-induced toxicity in $\mathrm{H} 9 \mathrm{c} 2$ cardiomyocytes and structural elucidation as 5-LOX inhibitors. Eur J Med Chem 180: 637-647, 2019.

13. Li H, Shi Y, Wang X, Li P, Zhang S, Wu T, Yan Y, Zhan Y, Ren Y, Rong X, et al: Piceatannol alleviates inflammation and oxidative stress via modulation of the Nrf2/HO-1 and NF- $\kappa \mathrm{B}$ pathways in diabetic cardiomyopathy. Chem Biol Interact 310: 108754, 2019.

14. Wang W, Yang R, Yao H, Wu Y, Pan W and Jia AQ: Inhibiting the formation of advanced glycation end-products by three stilbenes and the identification of their adducts. Food Chem 295: $10-15,2019$.

15. Moon I, Damodar K, Kim JK, Ryoo S and Jun JG: Synthesis, anti-inflammatory, and arginase inhibitory activity of piceatannol and its analogs. Bull Korean Chem Soc 38: 342-349, 2017.

16. Liu J, Zhou J, Wu Z, Wang X, Liu L and Yao C: Cyanidin 3-O- $\beta$-glucoside ameliorates ethanol-induced acute liver injury by attenuating oxidative stress and apoptosis: The role of SIRT 1/FOXO 1 signaling. Alcohol Clin Exp Res 40: 457-466, 2016.

17. Yan $X, Y u$, Zheng $H$, Wang $S, H e ~ Y$ and Wang $L$ : Calycosin-7-O- $\beta$-D-glucoside attenuates OGD/R-induced damage by preventing oxidative stress and neuronal apoptosis via the SIRT1/FOXO1/PGC-1 $\alpha$ pathway in HT22 cells. Neural Plast 2019: 8798069, 2019.

18. Llarena M, Andrade F, Hasnaoui M, Portillo MP, Pérez-Matute P, Arbones-Mainar JM, Hijona E, Villanueva-Millán MJ, Aguirre L, Carpéné C and Aldámiz-Echevarría L: Potential renoprotective effects of piceatannol in ameliorating the early-stage nephropathy associated with obesity in obese Zucker rats. J Physiol Biochem 72: 555-566, 2016.

19. Lee HJ, Kang MG, Cha HY, Kim YM, Lim Y and Yang SJ: Effects of piceatannol and resveratrol on sirtuins and hepatic inflammation in high-fat diet-fed mice. J Med Food 22: 833-840, 2019.

20. Fu Z, Yang J, Wei Y and Li J: Effects of piceatannol and pterostilbene against $\beta$-amyloid-induced apoptosis on the PI3K/Akt/Bad signaling pathway in PC12 cells. Food Funct 7: 1014-1023, 2016.

21. Wong CHY, Jenne CN, Lee WY, Léger C and Kubes P: Functional innervation of hepatic iNKT cells is immunosuppressive following stroke. Science 334: 101-105, 2011.

22. Longa EZ, Weinstein PR, Carlson S and Cummins R: Reversible middle cerebral artery occlusion without craniectomy in rats. Stroke 20: 84-91, 1989.

23. Paxinos G and Watson C: The Rat Brain in Stereotaxic Coordinates (Deluxe Edition). 4th edition. Rat Brain in Stereotaxic Coordinates, p256, 1998.

24. Xiong Y, Qu C, Mahmood A, Liu Z, Ning R, Li Y, Kaplan DL, Schallert T and Chopp M: Delayed transplantation of human marrow stromal cell-seeded scaffolds increases transcallosal neural fiber length, angiogenesis, and hippocampal neuronal survival and improves functional outcome after traumatic brain injury in rats. Brain Res 1263: 183-191, 2009.

25. Livak KJ and Schmittgen TD: Analysis of relative gene expression data using real-time quantitative PCR and the 2(-Delta Delta C(T)) method. Methods 25: 402-408, 2001.

26. Aebi H: Catalase in vitro. Methods Enzymol 105: 121-126, 1984.

27. Hafeman DG, Sunde RA and Hoekstra WG: Effect of dietary selenium on erythrocyte and liver glutathione peroxidase in the rat. J Nutr 104: 580-587, 1974.

28. Hissin PJ and Hilf R: A fluorometric method for determination of oxidized and reduced glutathione in tissues. Anal Biochem 74: 214-226, 1976 
29. Zhang W, Cui Y, Gao J, Li R, Jiang X, Tian Y, Wang K and Cui J: Recombinant osteopontin improves neurological functional recovery and protects against apoptosis via PI3K/Akt/GSK-3 $\beta$ pathway following intracerebral hemorrhage. Med Sci Monit 24: $1588-1596,2018$.

30. Kim HJ, Martemyanov KA and Thayer SA: Human immunodeficiency virus protein Tat induces synapse loss via a reversible process that is distinct from cell death. J Neurosci 28 12604-12613, 2008

31. Zhi G, Hai-Ping Z, Yu-Min L and Xun-Ming J: The behavioral testing in mice after cerebral ischemia. Chin J Comp Med 22 68-72, 2012.

32. Onwuekwe IO and Ezeala-Adikaibe B: Ischemic stroke and neuroprotection. Ann Med Health Sci Res 2: 186-190, 2012.

33. Rodrigo R, Fernández-Gajardo R, Gutiérrez R, Matamala JM, Carrasco R, Miranda-Merchak A and Feuerhake W: Oxidative stress and pathophysiology of ischemic stroke: Novel therapeutic opportunities. CNS Neurol Disord Drug Targets 12: 698-714, 2013.

34. Matsui Y, Sugiyama K, Kamei M, Takahashi T, Suzuki T, Katagata Y and Ito T: Extract of passion fruit (Passiflora edulis) seed containing high amounts of piceatannol inhibits melanogenesis and promotes collagen synthesis. J Agric Food Chem 58: 11112-11118, 2010

35. BanikK,Ranaware AM,HarshaC,NiteshT, GirisaS,Deshpande V Fan L, Nalawade SP, Sethi G and Kunnumakkara AB: Piceatannol: A natural stilbene for the prevention and treatment of cancer. Pharmacol Res 153: 104635, 2020.

36. Wen J, Lin H, Zhao M, Tao L, Yang Y, Xu X, Jia A, Zhang J and Weng D: Piceatannol attenuates D-GalN/LPS-induced hepatoxicity in mice: Involvement of ER stress, inflammation and oxidative stress. Int Immunopharmacol 64: 131-139, 2018.

37. Zhang Y, Zhang LH, Chen X, Zhang N and Li G: Piceatannol attenuates behavioral disorder and neurological deficits in aging mice via activating the Nrf2 pathway. Food Funct 9: 371-378, 2018.

38. Kinoshita Y, Kawakami S, Yanae K, Sano S, Uchida H, Inagaki $\mathrm{H}$ and Ito $\mathrm{T}$ : Effect of long-term piceatannol treatment on eNOS levels in cultured endothelial cells. Biochem Biophys Res Commun 430: 1164-1168, 2013.

39. Kiliç V: Piceatannol mediated modulation of oxidative stress and regeneration in the liver of endotoxemic mice. J Med Food 22: 594-601, 2019.

40. Piotrowska H, Kucinska M and Murias M: Biological activity of piceatannol: Leaving the shadow of resveratrol. Mutat Res 750: 60-82,2012.
41. Yokozawa T and Kim YJ: Piceatannol inhibits melanogenesis by its antioxidative actions. Biol Pharm Bull 30: 2007-2011, 2007.

42. Kim HJ, Lee KW and Lee HJ: Protective effects of piceatannol against beta-amyloid-induced neuronal cell death. Ann N Y Acad Sci 1095: 473-482, 2007.

43. Song H, Jung JI, Cho HJ, Her S, Kwon SH, Yu R, Kang YH, Lee KW and Park JHY: Inhibition of tumor progression by oral piceatannol in mouse 4T1 mammary cancer is associated with decreased angiogenesis and macrophage infiltration. J Nutr Biochem 26: 1368-1378, 2015.

44. Wen H, Fu Z, Wei Y, Zhang X, Ma L, Gu L and Li J: Antioxidant activity and neuroprotective activity of stilbenoids in rat primary cortex neurons via the PI3K/Akt signalling pathway. Molecules 23: 2328, 2018.

45. Hong KS, Park JI, Kim MJ, Kim HB, Lee JW, Dao TT, Oh WK, Kang CD and Kim SH: Involvement of SIRT1 in hypoxic down-regulation of c-Myc and $\beta$-catenin and hypoxic preconditioning effect of polyphenols. Toxicol Appl Pharmacol 259: 210-218, 2012.

46. Jiang Y, Luo W, Wang B, Wang $\mathrm{X}$, Gong $\mathrm{P}$ and Xiong $\mathrm{Y}$ : Resveratrol promotes osteogenesis via activating SIRT1/FoxO1 pathway in osteoporosis mice. Life Sci 246: 117422, 2020.

47. Kitada M, Ogura Y, Maruki-Uchida H, Sai M, Suzuki T, Kanasaki K, Hara Y, Seto H, Kuroshima Y, Monno I and Koya D: The effect of piceatannol from passion fruit (Passiflora edulis) seeds on metabolic health in humans. Nutrients 9: 1142, 2017.

48. Arai D, Kataoka R, Otsuka S, Kawamura M, Maruki-Uchida H, Sai M, Ito T and Nakao Y: Piceatannol is superior to resveratrol in promoting neural stem cell differentiation into astrocytes. Food Funct 7: 4432-4441, 2016.

49. Suzuki Y, Nakano Y, Mishiro K, Takagi T, Tsuruma K, Nakamura M, Yoshimura S, Shimazawa $M$ and Hara $H$ : Involvement of Mincle and Syk in the changes to innate immunity after ischemic stroke. Sci Rep 3: 3177, 2013.

50. Ye XC, Hao Q, Ma WJ, Zhao QC, Wang WW, Yin HH, Zhang T, Wang M, Zan K, Yang XX, et al: Dectin-1/Syk signaling triggers neuroinflammation after ischemic stroke in mice. J Neuroinflammation 17: 17, 2020.

This work is licensed under a Creative Commons

Attribution-NonCommercial-NoDerivatives 4.0 International (CC BY-NC-ND 4.0) License. 